\title{
IMPACTO DO TRATAMENTO COM REALIDADE VIRTUAL NO RISCO DE QUEDAS EM IDOSOS
}

Gustavo Willames Pimentel BARROS

Luís Roberto Gomes da SILVA

Walmir Romário dos SANTOS ${ }^{2}$

Hugo TOURINHO FILHO ${ }^{3}$

Wlaldemir Roberto dos SANTOS $^{4}$

\begin{abstract}
${ }^{1}$ Graduado em Educação Física, Departamento de Educação Física da Universidade Federal de Pernambuco, Recife, Pernambuco, Brasil.

${ }^{2}$ Graduando em Educação Física, Escola de Educação Física e Esporte de Ribeirão Preto da Universidade de São Paulo, Ribeirão Preto, São Paulo, Brasil.

${ }^{3}$ Graduado em Educação Física, Mestre em Ciência do Movimento Humano, Doutor em Educação Física, Docente da Escola de Educação Física e Esporte de Ribeirão Preto, São Paulo, Brasil.

${ }^{4}$ Graduado em Educação Física e Fisioterapia, Mestre em Ciências, Doutorando em Ciências Biológicas, Centro de Ciências da biológica da Universidade Federal de Pernambuco, Recife, Pernambuco, Brasil, wlaldemir@hotmail.com
\end{abstract}

Recebido em: 25/07/2015 - Aprovado em: 08/01/2016 - Disponibilizado em: 30/07/2016

RESUMO: Objetivo: verificar o impacto do treinamento com realidade virtual no risco de quedas em idosos. Métodos: a amostra foi composta por 10 sujeitos, do sexo feminino (com idade de 55,80 $\pm 5,90$ anos, estatura de 154,00 $\pm 0,07$ cm e massa corporal de $64,15 \pm 13,22 \mathrm{~kg}$ ). Para a avaliação dos indivíduos foi utilizada a escala de Berg e o índice dinâmico de marcha, antes e após às nove sessões de treinamento em vídeo game ativo (realizado com o vídeo game Xbox, com Kinect e o jogo Kinect Adventures da Microsoft $\left.{ }^{\circledR}\right)$. O teste de normalidade Shapiro-Wilkapontou normalidade dos dados em todas as comparações realizadas. No que se refere a investigação intragrupos, o teste $\mathrm{T}$ pareado de Student foi utilizado. Todas as análises foram realizadas pelo SPSS 17.0 com nível de significância previamente estabelecido ( $\mathrm{p}<0,05)$. Resultados: A análise comparativa apontou um aumento significativo na pontuação de ambos os testes (Escala de Berg - p=0,002 e o Índice Dinâmico de Marca $-\mathrm{p}=0,001$ ). Conclusão: Os resultados apontam que a realidade virtual pode ser utilizada na redução do risco de queda em idosos. Podendo, assim, servir como recurso complementar para reduzir os efeitos deletérios do envelhecimento.

DESCRITORES: Envelhecimento; Quedas; Realidade Virtual; Capacidade Motora; Qualidade de Vida.

\section{IMPACT OF TREATMENT WITH VIRTUAL REALITY AT RISCK OF FALLS IN ELDERLY}

\begin{abstract}
Objective: To investigate the impact of training with virtual reality in the risk of falls in the elderly. Methods: The sample consisted of 10 subjects, female (aged 55.80 \pm 5.90 years, height of $154.00 \pm 0.07 \mathrm{~cm}$ and body mass $64.15 \pm 13.22 \mathrm{~kg}$ ). For the evaluation of individuals we used the Berg scale and the dynamic gait index before and after nine sessions of training in active video game (done with the video game Xbox with Kinect and Kinect Adventures Microsoft ${ }^{\circledR}$ game). The normality test Shapiro-Wilk normality of the data pointed in all comparisons. Concerning the intra-group research, paired Student t-test was used. All analyzes were performed using SPSS 17.0 with previously established level of significance $(p<0.05)$. Results: Comparative analysis showed a significant increase in scores of both tests (Berg scale $-p=0.002$ and the Dynamic Index mark $-p=0.001$ ). Conclusion: The results show that virtual reality can be used to reduce the risk of falls in the elderly. And thus can serve as a complementary resource to reduce the deleterious effects of aging.
\end{abstract}

KEY WORDS: Aging; Falls; Virtual Reality; Motor capacity; Quality of life. 


\section{INTRODUÇÃO}

$\mathrm{Na}$ medida em que o ser humano envelhece aumenta a predisposição a quedas, tornando-se o principal problema de cuidado a saúde da população idosa (CASTRO et al., 2015). Cerca de 30\% dos indivíduos acima dos 65 anos sofrem quedas ao menos uma vez por ano (SIQUEIRA et al., 2007), aumentando os gastospúblicoscom os problemas decorrentes das quedas (JORGE; KOIZUMI, 2004). Entre as principais consequências das quedas, destacam-se as fraturas, aumento de dependência, morbidades e mortalidades (CUNHA; LOURENÇO, 2014). No mais, as quedas podem influenciar diretamente na rotina de cuidadores e familiares, devido aos cuidados especiais exigidos pela recuperação ou adaptação dos idososapós a queda (SIQUEIRA et al., 2007).

Diversos fatoresrelacionados ao risco de queda em idosos são citados pela Organização Mundial da Saúde (KALACHE, 2010), como redução da força muscular, alterações visuais, redução do equilíbrio, alterações de marcha e ortopédicas, déficits cognitivos, efeitos adversos de medicamentos, fatores ambientais, comportamentais e sociais. Segundo Cunha e Lourenço (2014) o principal fator que aumenta o risco de queda em idosos é aalteração no ciclo da marcha que ocorre no decorrer da idade, associado a redução do equilíbrio, que em indivíduos acima dos 65 anos pode chegar a 85\% (GARCIA, 2011).

Sendo assim, minimizar os efeitos deletérios do envelhecimento, principalmente o déficit de equilíbrio e da marcha, torna-se de extrema importância na redução do risco de queda em idosos,consequentemente, reduzindo o número de morbidade $\mathrm{e}$ mortalidades, impactando na qualidade de vida e longevidade de idosos (FRANCHI; MONTENEGRO, 2005). Entre as estratégias utilizadas para minimizar os efeitos do envelhecimento, o uso de terapias lúdicas vem sendo bastante utilizado, auxiliando na aderência e participação dos idosos aos exercícios propostos (FLEURÍ et al., 2013).

Atualmente a terapia de exposição à realidade virtual, a qual ocorre em uma interface entre o paciente e o vídeo game que simula o ambiente real, através da interação dos sinais gráficos com canais sensoriais e motores, vem se destacando como ferramenta auxiliar na reabilitação de diversos pacientes, destacando-se benefícios como a correção do equilíbrio, postura, melhora na marcha, funcionalidade e autoestima (SCHIAVINATO et al., 2010).

Neste sentido, devido à dificuldade da marcha e ao déficit do equilíbrio associado ao risco aumentado de quedas em idosos, ao grande número de morbidade e mortalidade e ao uso do tratamento com realidade virtual na reabilitação da marcha e do equilíbrio, o presente estudo teve como objetivo verificar 
o impacto do treinamento com realidade virtual no risco de quedas em idosos.

\section{METODOS}

\section{Sujeitos e desenho do estudo}

A amostra foi composta por dez sujeitos, do sexo feminino, tendo as suas características descritas na Tabela 1. Os sujeitos foram convidados a participar voluntariamente do estudo, sendo informados sobre todos os riscos e benefícios. Foram incluídos os que concordaram em participar assinando o termo de consentimento livre e esclarecido, que estavam engajados em um projeto de pesquisa com idosos realizados no Departamento de Educação Física da Universidade Federal de Pernambuco no Recife/PE e que não possuíam qualquer enfermidade que pudesse comprometer as respostas cardiovasculares e/ou limitações articulares que impossibilitassem a realização dos exercícios. Os procedimentos deste estudo seguiram todas as normas éticas estipuladas de acordo com a resolução 196/96 que regulamenta a pesquisa envolvendo seres humanos. Após a seleção dos participantes, de acordo com os critérios supracitados, os sujeitos foram submetidos ao teste de equilíbrio de Berg e ao índice do dinâmico de marcha, para verificaro risco de quedas. Na sequência, os sujeitos foram submetidos ao protocolo de treinamento com a realidade virtual, no qual foi utilizado um vídeo game Xbox, por nove sessões de treinamentos, sendo três vezes por semana, com duração de 15 minutos cada sessão, totalizando três semanas de treinamento. Ao término do protocolo de treinamento, os sujeitos tiveram o Teste de equilíbrio de Berg e ao índice dinâmico de marcha repetidos.

\section{Caracterização da amostra}

A idade, estatura e a massa corporal total dos sujeitos foram verificadas a partir do cadastro pré-existente dos sujeitos no projeto em que todos eram inseridos.

\section{Teste de equilíbrio de Berg e índice dinâmico de marcha}

O teste de equilíbrio de Berg foi descrito por Katherine Berg em 1992 e adaptada para a língua portuguesa por Miyamoto et al. (2004). O objetivo do teste de Berg é determinar o equilíbrio e, assim, verificar o risco de quedas. $O$ teste é composto por atividades de vida diária, baseada em 14 itens, onde cada item possui cinco alternativas que variam de zero a quatro pontos, sendo zero a inabilidade de desenvolver a ação solicitada e quatro a habilidade total de exercer a função. Sendo assim, a pontuação do teste é de 56 pontos, onde um valor menor de 45 pontos é considerado preditor de queda e um índice menor ou igual a 36 pontos está associado a $100 \%$ de risco de queda (HAYES; JOHNSON, 2003; MIYAMOTO et al., 2004; SILVA et al., 2008). 
O índice dinâmicode marcha foi desenvolvido por Shumway-Cook et al. em 1997, tendo o intuito de avaliar o equilíbrio dinâmico e o risco de quedas (CASTRO et al., 2006) O índice consiste em testes dinâmicos que incentivam estímulos vestibulares durante a marcha com obstáculo, sendo composto por oito itens com quatro alternativas, que variam de zero a três pontos, onde zero indica grave comprometimento e três indica desempenho normal da função. Assim, o índice possuiu pontuação máxima de 24 pontos, tendo indicativo de risco de queda quando o índice for menor ou igual a 19 pontos (ROGATTO et al., 2010).

\section{Vídeo game Xbox}

O tratamento com realidade virtual foi realizado com o vídeo game Xbox, com Kinect e o jogo Kinect Adventuresda Microsoft $\AA$, consistindo por nove sessões, sendo três vezes por semana, com duração de 15 minutos cada sessão, totalizando três semanas de treinamento. Todas as sessões de treinamentos foram realizadas as 8:00 e as 14:00, sendo os sujeitos divididos igualmente entre os dois períodos nas segundas, quartas e sextas-feiras. Assim,tendo de 48 a 72 horas de intervalo entre as sessões.

\section{Análise estatística}

Para interpretação dos dados, a análise descritiva foi empregada para caracterização da amostra expressa por valores de média e desvio padrão. Inicialmente um teste exploratório indicou inexistência de "outliers". O teste de normalidade (Shapiro-Wilk) indicou normalidade dos dados em todas as comparações. Para as comparações intragrupos (antes e após o treinamento) o teste $\mathrm{t}$ pareado foi empregado. Todas as análises foram realizadas no software StatisticalPackage for the Social Sciences SPSS 17.0 com nível de significância previamente estabelecido $(p<0,05)$.

\section{RESULTADOS}

Avaliou-se dez sujeitos, sendo todas mulheres, com suas características descritas na Tabela 1.

Tabela 1- Caracterização da amostra.

\begin{tabular}{lc}
\hline \multicolumn{1}{c}{ Variável } & Média \pm Desvio Padrão \\
\hline Idade (anos) & $55,80 \pm 5,90$ \\
Estatura $(\mathrm{cm})$ & $154,00 \pm 0,07$ \\
Massa corporal total $(\mathrm{kg})$ & $64,15 \pm 13,22$ \\
\hline
\end{tabular}

Fonte: dos autores

Na média dos testes realizados houve aumento significante no período pré e pós treinamento de realidade virtual que foi realizado por três semanas (nove sessões). No teste de equilíbrio de Berg se verificou um aumento médio dos scores de $13,70 \%(p=0,002)$. Em relação ao índice dinâmico da marcha, a média dos scores aumentou em $19,53 \%(p=0,001)$. 
Tabela 2 -Teste de equilíbrio de Berg e índice dinâmico de marcha antes e após o protocolo de treinamento da Realidade Virtual.

\begin{tabular}{|c|c|c|c|}
\hline Testes & $\begin{array}{c}\text { Pré- } \\
\text { treino } \\
\text { Score } \\
\text { médio } \pm \\
\text { DP } \\
\text { (min- } \\
\text { máx) }\end{array}$ & $\begin{array}{c}\text { Pós- } \\
\text { treino } \\
\text { Score } \\
\text { médio } \pm \\
\text { DP } \\
\text { (min- } \\
\text { máx) }\end{array}$ & $\begin{array}{c}\text { Diferença } \\
\text { média entre } \\
\text { os Scores } \\
(\%)\end{array}$ \\
\hline $\begin{array}{l}\text { Teste de } \\
\text { equilíbrio } \\
\text { de Berg }\end{array}$ & $\begin{array}{c}37,5 \pm 6,29 \\
(25-45)\end{array}$ & $\begin{array}{c}42.7 \pm 3,83 \\
\quad(37-50)\end{array}$ & $\begin{array}{c}5,2(13,70 \%) \\
0,002 *\end{array}$ \\
\hline $\begin{array}{l}\text { Índice } \\
\text { dinâmico } \\
\text { de marcha }\end{array}$ & $\begin{array}{c}16,9 \pm \\
2,84 \\
(12-20)\end{array}$ & $\begin{array}{c}20.2 \pm \\
1,68 \\
(17-22)\end{array}$ & $\begin{array}{c}3,3(19,53 \%) \\
0,001 *\end{array}$ \\
\hline
\end{tabular}

Fonte: dos autores

\section{DISCUSSÃO}

Os jogos selecionados para este estudo oferecem desequilíbrios látero-lateral e ântero-posterior e, assim, estimulam o sistema proprioceptivos que,auxiliado pelo feedbackvisual,leva a melhora do equilíbrio. SegundoDefarini, Rocha e Brandão(2012) os jogos do Kinect Adventures proporcionam aos seus praticantes melhor resistência, coordenação motora e equilíbrio, impactando diretamente na marcha, consequentemente, reduzindo o risco de quedas.

No presente estudo, verificou-se aumento significante nos scores médios do teste de equilíbrio de Berg e do índice dinâmico de marcha, mostrando que, em um curto período de treinamento de realidade virtual (nove sessões - três semanas), o risco de queda foi reduzido.
Os resultados corroboram com Pigford e Andrews (2010), que verificou redução do risco de quedas em idosos, utilizando o Wii Fitß. Em pacientes com disfunção cerebelar, Schiavinatoet al. (2011), verificou redução no risco de queda, utilizando Wii Fit ${ }^{\circledR}$ combinada com outras técnicas fisioterapêuticas.

Protocolos utilizando Wii Fit®vem sendo utilizando na literatura mostrando melhora do equilíbrio, capacidade funcional e ajustes posturais (GAZZOLA et al., 2009; TEIXEIRA et al., 2010; BARCALA et al., 2011), que também foi verificada por Keshnere Kenyon(2004) utilizando um sistema de ambiente virtual com uma plataforma em terceira dimensão e por Borges e Mendes (2015) utilizando o Xbox.Segundo You (2005) as melhoras citadas nos estudos acima, podem estar relacionadascom melhor tempo de reação, velocidade e controle direcionais dos movimentos. Melhoras que também pode ter sido associada ao presente estudo, uma vez que os testes necessitam do equilíbrio (dinâmico e estático), função, postura e mudanças de direção.

Em outro estudo, o Wii Fit ${ }^{\circledR}$ promoveu aumento significante do equilíbrio e força muscular em 10 mulheres saudáveis, com idades entre 30 e 60 anos, quando as mesmas foram submetidas a sessões de 30 minutos, 2 vezes por semana, durante 10 semanas (NITZ, 2010).Segundo 
Ribeiro et al (2009), as alterações associadas ao envelhecimento, tais como declínio da massa muscular, são fatores importantes associados a redução da mobilidade funcional e equilíbrio, proporcionando, assim, um maior risco de quedas. Utilizando oXbox o presente estudo verificou a redução no risco de quedas, que pode ter sido acompanhado de melhora da força muscular dos sujeitos, entretanto, a variável força não foi verificada no presente estudo.

\section{REFERÊNCIAS}

BARCALA, Luciana et al. Análise do equilíbrio em pacientes hemiparéticos após o treino com o programa Wii Fit. Fisioterapia em Movimento, v. 24, n. 2, p. 337-343, 2011.

BORGES, Ana Paula Mazzarino; MENDES, Giorgia Caroline. Avaliação cognitiva e de equilíbrio em idosos institucionalizados após intervenção de xbox terapia. Caderno da Escola de Saúde, v. 1, n. 13, 2015.

CASTRO, Paula Maria Machado Arantes et al. Testes de equilíbrio e mobilidade funcional na predição e prevenção de riscos de quedas em idosos. Revista Brasileira de Geriatria e Gerontologia, v. 18, n. 1, p. 129-140, 2015.

CUNHA, Alfredo; LOURENÇO, Roberto. Quedas em idosos: prevalência e fatores associados. Revista Hospital Universitário Pedro Ernesto, v. 13, n. 2, 2014.

DEFAVARI, Pollyeverlin R; ROCHA, Alex H.; BRANDÃO, Pierre S. Estudo da viabilidade da utilização do Kinect como ferramenta no atendimento fisioterapêutico de pacientes neurológicos. XI SBGames, v. 1, n.1, 2012.

\section{CONCLUSÃO}

Diante disto, sugerimos que o tratamento comrealidade virtual pode ser utilizado na redução do risco de queda em idosos. Podendo, assim, servir como recurso complementar para reduzir os efeitos deletérios do envelhecimento. Porém, cabe ressaltar, a carência de estudos sobre o tema, o que encoraja a realização de pesquisas futuras. Além da necessidade de elucidar de forma mais significante os resultados encontrados neste estudo, com um número maior de pacientes.

FLEURÍ, Amanda Caroline P. et al. Atividades lúdicas com idosos institucionalizados. Enfermagem Revista, v. 16, n. 1, p. 50-57, 2013.

FRANCHI, Kristiane Mesquita Barros; MONTENEGRO, Renan Magalhães. Atividade física: uma necessidade para a boa saúde na terceira idade. Revista Brasileira em Promoção da Saúde, v. 18, n. 3, p. 152156, 2005.

GARCIA, Patrícia A. et al. Estudo da relação entre função muscular, mobilidade funcional e nível de atividade física em idosos comunitários. Revista Brasileira de Fisioterapia, v. 15, n. 1, p. 15-22, 2011.

GAZZOLA, Juliana Maria et al. Realidade virtual na avaliação e reabilitação dos distúrbios vestibulares. Acta ORL/ Técnicas e Otorrinolaringologia, v. 27, n. 1, p. 22-7, 2009.

HAYES, Karen. W.; JOHNSON, Marjorie. E. Berg balance scale. American CollegeofRheumatology, v. 4, p. 28-30, 2003.

JORGE, Maria Helena Prado de Mello; KOIZUMI, Maria Sumie. Gastos governamentais do SUS com internações hospitalares por causas externas: análise no 
Estado de São Paulo, 2000. Revista Brasileira de Epidemiologia, v. 7, n. 2, p. 228-238, 2004.

KALACHE, A. Relatório Global da OMS sobre Prevenção de Quedas na velhice. São Paulo, 2010.

KESHNER, Emily A.; KENYON, Robert V. Usingimmersivetechnology for postural researchandrehabilitation. Assistive

Technology, v. 16, n. 1, p. 54-62, 2004.

MIYAMOTO, Sayuri. et al. Brazilianversionofthe Berg balance scale. BrazilianJournalof Medical andBiologicalResearch, v. 37, n. 9, p. 14111421, 2004.

NITZ, Jennifer. C. et al. Isthe Wii Fit ${ }^{\mathrm{TM}}$ a new-generation tool for improving balance, healthandwell-being?

pilotstudy. Climacteric, v. 13 , n. 5, p. 487491, 2010.

PIGFORD, Thomas.; ANDREWS, A. Williams. Feasibilityandbenefitofusingthe Nintendo Wii Fit for balance rehabilitation in anelderlypatientexperiencingrecurrentfalls. J ournalofStudentPhysicalTherapyResearch , v. 2, n. 1, p. 12-20, 2010.

RIBEIRO, Fernando et al. Impacto da prática regular de exercício físico no equilíbrio, mobilidade funcional e risco de queda em idosos institucionalizados. Revista Portuguesa de Ciências do Desporto, v. 9, n. 1, p. 36-42, 2009.

ROGATTO, Adriana Roberta Degressi et al. Proposta de um protocolo para reabilitação vestibular em vestibulopatias periféricas. Fisioterapia em Movimento, v. 23, n. 1, p. 83-91, 2010.

SCHIAVINATO, Alessandra $M$. et al. Influência da realidade virtual no equilíbrio de paciente portador de disfunção cerebelarEstudo de caso. Revista Neurociência, v. 19, n. 1, p. 119-127, 2011.

SCHIAVINATO, Alessandra $M$. et al. Influência do Wii Fit no equilíbrio de paciente com disfunção cerebelar: estudo de caso. Journalof Health Science Institute, v. 28, n. 1, p. 50-2, 2010.

SILVA, Andressa da et al. Equilíbrio, coordenação e agilidade de idosos submetidos à prática de exercícios físicos resistidos. Revista Brasileira de Medicina do Esporte, v. 14, n. 2, p. 88-93, 2008.

SIQUEIRA, Fernando V. et al. Prevalência de quedas em idosos e fatores associados. Revista Saúde Pública, v. 41, n. 5, p. 749-56, 2007.

TEIXEIRA, Clarissa Stefani et al. Sistema estomatognático postura e equilíbrio corporal. Salusvita, Bauru, v. 29, n. 2, p. 6981, 2010.

YOU, Sung H. et al. Virtual reality-induced cortical reorganizationandassociated locomotor recovery in chronicstrokeanexperimenterblindrandomizedstudy. Stroke, v. 36, n. 6, p. 1166-1171, 2005. 